\title{
Editorial: From the Fermi Scale to Cosmology
}

\author{
Alberto Salvio ${ }^{1 *}$ and Francesco Sannino ${ }^{2}$ \\ ${ }^{1}$ Physics Department, University of Rome, INFN Tor Vergata, Rome, Italy, ${ }^{2}$ CP3-Origins, University of Southern Denmark, \\ Odense, Denmark
}

Keywords: standard model and beyond, cosmology-theory, gravitation, field theory, astrophysics

\section{Editorial on the Research Topic}

\section{From the Fermi Scale to Cosmology}

In July 2012 (Aad et al., 2012; Chatrchyan et al., 2012) the Large Hadron Collider (LHC) experimental collaborations ATLAS and CMS announced the discovery of the Higgs boson, the long sought elementary particle of the Standard Model (SM) of particle interactions. The discovery crowned the SM as the most successful description of natural phenomena at the shortest distance ever tested by humans.

The discovery left still a number of unanswered questions since the SM remains an incomplete description of nature. In fact, it fails to account for neutrino masses, the particle nature of dark matter (DM) and last but not the least the observed asymmetry between matter and antimatter in the universe (BAU).

Intriguingly, some of the issues above, specifically DM and BAU, lie at the interface between particle physics (encoded in the SM) and cosmology. This has naturally intensified the efforts to arrive at a deeper understanding of the connection between the physics of very short distances and the one dealing with very large ones. For example, the precise knowledge of the SM parameters allows us to make sensible predictions in cosmology.

Of course, in order for this to be feasible the SM should be embedded in a theory of gravity. Einstein's theory, a.k.a General Relativity (GR), is extremely successful and able to describe gravity and its interactions with matter from the largest scales almost down to the extremely small Planck length $l_{P} \sim 10^{-33} \mathrm{~cm}$. However, when approaching $l_{P}$, GR cannot be treated classically and needs to be quantized. If we were to naively quantize GR one would discover that it is not renormalizable, like the pion Lagrangian. This fact tells us that it is only a low energy description of physical phenomena and that below or at around the Planck length the true elementary nature will appear. Currently a satisfactory quantum theory of gravity is still missing and both theoretical and experimental inputs are needed to determine its nature.

The purpose of the Research Topic "From the Fermi Scale to Cosmology" is to bring to light the latest exciting ideas and results that are meant to elucidate the relation between the LHC collider program and astrophysics, paying attention to their impact on cosmology.

Perhaps a natural subject along these lines is the potential cosmological role of the Higgs boson. In Bezrukov and Shaposhnikov $(2008,2014)$, it was proposed that the Higgs could play the role of the inflaton. This remains an interesting possibility and therefore Rubio provided an in depth overview on this subject. Although this is an economical and predictive model of the early universe its viability is intimately related to the nature of the electroweak vacuum in the SM. Its fate is not yet sealed since it could still be absolutely stable, unstable, or metastable with a preference for the last case according to the latest determinations of the top mass $m_{t}$. It is for this reason that in recent years much work has been devoted to understand whether the metastable case can yield a consistent cosmology and, if so, what are the physical implications. A contribution 
by Markkanen et al. presents a comprehensive review of the implications of the Higgs vacuum metastability for cosmology along with a pedagogical discussion of the related theoretical topics, including renormalization group improvement, quantum field theory in curved spacetime and vacuum decay in field theory.

However, it is still possible, although unlikely, that we do not leave in a metastable vacuum, but rather in an absolutely stable one because the uncertainty on $m_{t}$ is still fairly large. Corcella, in his review on the definition and determination of $m_{t}$, discusses the main strategies to extract $m_{t}$ at the LHC and the interpretation of the measurements in terms of well-posed top-mass definitions, taking particular care about renormalon ambiguities, progress in Monte Carlo event generators for top physics and theoretical uncertainties.

Of course, even if we were certain about the fate of the SM vacuum, it would still be possible (and actually likely) that new degrees of freedom (elementary particles) with masses below the Planck mass are present in nature. In this case, many particle physics models of the early universe can be investigated. An interesting possibility is that the inflaton is identified with the radial scalar of an axion model. The axion (Weinberg, 1978; Wilczek, 1978) is independently motivated by the strong CP problem (Peccei and Quinn, 1977a,b) and is a viable DM candidate. Ballesteros et al. contribute to this Research Topic with an overview on this possibility, which, along the lines of a previous work (Salvio, 2015), is combined with a model of neutrino masses that is capable of accounting for BAU.

About DM, Arina explains the impact of cosmological and astrophysical constraints on DM, focusing on simplified models. She classifies the models that have been analyzed so far and for each of them she reviews in detail the complementarity of relic density, direct, and indirect searches with respect to the LHC searches. She also discusses the capabilities of each type of search to identify regions where individual approaches to dark matter detection are the most relevant to constrain the model parameter space. Finally, a critical overview on the validity of the dark matter simplified models is provided and the caveats for the interpretation of the experimental results extracted for these models are discussed.

As mentioned above, besides DM, neutrino masses furnish further evidence for BSM. One of the currently most exciting research areas regarding neutrino masses is about their ordering. de Salas et al. provide us with a review on the status of our understanding of neutrino mass ordering and future prospects. Quite interestingly the Bayesian analysis to the 2018 publicly

\section{REFERENCES}

Aad, G., Abajyan, T., Abbott, B., Abdallah, J., Abdel Khalek, S., Abdelalim, A. A., et al. (2012). Observation of a new particle in the search for the Standard Model Higgs boson with the ATLAS detector at the LHC. Phys. Lett. B 716, 1-29. doi: 10.1016/j.physletb.2012.08.020

Bezrukov F., and Shaposhnikov, M. (2014). Higgs inflation at the critical point. Phys. Lett. B 734, 249-254. doi: 10.1016/j.physletb.2014.05.074 available oscillation and cosmological data sets provides strong evidence for the normal neutrino mass ordering vs. the inverted scenario, with a significance of 3.5 standard deviations.

Beyond experimental facts theoretical considerations further motivate extending or modifying the SM and GR. For example the lack of predictivity of GR at length scales smaller than $l_{P}$ calls for new ideas to address this issue. Focusing on scenarios that maintain the GR relativistic field theoretic structure while remaining in four dimensions, this editorial features two reviews [written by Eichhorn and Salvio (one of the editors of this Research Topic)] on the recent efforts to address the lack of GR predictibility. Both approaches essentially require gravitational and non-gravitational interactions to flow to a UV fixed point. Eichhorn's review focuses on Weinberg's asymptotic safety (Weinberg, 1977, 1980) where an infinite number of terms are added to the Einstein-Hilbert action to ensure renormalizability. Only very recently asymptotic safety has been rigorously established in four dimensions for gaugeYukawa theories (Litim and Sannino, 2014). A complementary point of view is described in Salvio's review of quadratic gravity (adding only the terms quadratic in the curvature to the EinsteinHilbert action renders gravity renormalizable).

In all these scenarios, as well as in GR, non-perturbative gravitational effects, known as gravitational instantons, can have deep implications for both particle physics and cosmology. A review by Hebecker et al. revisits some of the more recent discussions of the phenomenological relevance of gravitational instantons. In particular, these instantons are expected to break the shift symmetries of axions or Goldstone bosons non-perturbatively.

Clearly the subjects of SM physics and beyond, quantum gravity and the interplay with cosmology are vast and this Research Topic is by no mean meant as an exhaustive review. The choice of the topics reflect partially our interests and partially the limited time we had at our disposal for a more in depth review. Additionally, we also wished to guide the reader through a less dispersive choice of arguments. We sincerely hope that this Research Topic will inspire the readers to tackle and solve many of the exciting unsolved issues discussed by our colleagues in their reviews. Finally, it is our pleasure to thank the colleagues that accepted being part of this effort for their excellent contributions.

\section{AUTHOR CONTRIBUTIONS}

All authors listed have made a substantial, direct and intellectual contribution to the work, and approved it for publication. 
Peccei R. D., and Quinn, H. R. (1977a). CP conservation in the presence of instantons. Phys. Rev. Lett. 38:1440. doi: 10.1103/PhysRevLett.38.1440

Peccei R. D., and Quinn, H. R. (1977b). Constraints imposed by CP conservation in the presence of instantons. Phys. Rev. D 16, 1791-1797. doi: 10.1103/PhysRevD.16.1791

Salvio, A. (2015). A simple motivated completion of the standard model below the planck scale: axions and right-handed neutrinos. Phys. Lett. B 743 428-434. doi: 10.1016/j.physletb.2015.03.015

Weinberg, S. (1978). A new light Boson? Phys. Rev. Lett. 40:223. doi: 10.1103/PhysRevLett.40.223

Weinberg, S. (1977). Understanding the Fundamental Constituents of Matter. ed A. Zichichi. New York, NY: Plenum Press.

Weinberg, S. (1980). General Relativity: An Einstein Centenary Survey. eds S. W. Hawking and W. Israel (Cambridge: Cambridge University Press), 790-831.
Wilczek, F. (1978). Problem of strong $P$ and $T$ invariance in the presence of instantons. Phys. Rev. Lett. 40:279. doi: 10.1103/PhysRevLett. 40.279

Conflict of Interest Statement: The authors declare that the research was conducted in the absence of any commercial or financial relationships that could be construed as a potential conflict of interest.

Copyright $\odot 2019$ Salvio and Sannino. This is an open-access article distributed under the terms of the Creative Commons Attribution License (CC BY). The use, distribution or reproduction in other forums is permitted, provided the original author(s) and the copyright owner(s) are credited and that the original publication in this journal is cited, in accordance with accepted academic practice. No use, distribution or reproduction is permitted which does not comply with these terms. 